\title{
THE BASIC PRINCIPLES OF SYSTEMS THINKING AND SYSTEM DYNAMICS
}

\author{
Dr Brian Dangerfield \\ dangerfiel@gmail.com
}

\begin{abstract}
This tutorial paper presents the basics of System Dynamics (SD) modelling, together with an introductory section on Systems Thinking, specifically influence (or causal loop) diagrams. The coverage of SD starts with how a stock-flow diagram is used to commence the conceptualisation process by the creation of a spinal flow(s). Auxiliary variables (contained in the information system) and model parameters are then deployed in equations which explain the various flow rates depicted in the spinal flows. By this means a full working model emerges with a web of information overlaid onto the spinal flows. The paper concludes with a fully worked (but simple) example in the domain of workforce modelling.
\end{abstract}

Keywords: Systems Thinking; System Dynamics; Influence Diagram; Stock-flow Diagram.

\section{INTRODUCTION}

This tutorial paper introduces the basics of the system dynamics simulation methodology, together with the adjunct field of systems thinking which emerged subsequently. The field of system dynamics was initially known as Industrial Dynamics which reflected its origins in the simulation of industrial supply chain problems. The first paper published by the founder of the field, Jay W Forrester, appeared in 1958 (Forrester, 1958) and it was a precursor to what proved to be a hugely influential book: Industrial Dynamics (1961). Forrester sought to apply concepts of control engineering to management type problems and was very probably influenced by the earlier work of Arnold Tustin (1953). Forrester argued that the field of Operations/Operational Research (OR) at that time was not focused on the sort of problems that sought to inform policy (top-level) issues in an organisation. By its very definition OR was restricted to operational problems. Forrester saw a niche for a methodology which could tackle strategic issues more appropriately addressed to the success or failure of an organisation, as well as prominent national and international policy issues. See Forrester (2007) for his personal recollections of the history of the field.

The characteristics of system dynamics simulation models can be listed as follows:

- They address issues by considering aggregates (of products, people etc) and not individual entities (as in discrete event simulation) or individual agents (as in agent based modelling)

- They primarily reflect the dynamics of a system as having endogenous causes: change over time comes from within the system boundary due to information feedback effects and component interactions, although the initial stimulus for those dynamics may be exogenous. For more on the endogenous perspective see Richardson (2011).

- They carefully distinguish between resource flows and the information flows which cause those resource flows to increase or run down. This is a fundamental (and powerful) feature of the methodology which means such models can be used to design and evaluate information systems as well as the more usual focus on resource systems. 


\section{Dangerfield}

- The flows are assumed to be continuous and are governed by what are in effect ordinary differential equations. System dynamics models belong to the broader category of continuous simulation models.

- Although flow rates are included, SD models are primarily concerned with the behaviour of stocks or accumulations in the system. These are described by integral equations. Forrester has famously stated that differentiation does not exist in nature, only integration.

Mathematical models characterised by differential equations must be solved in order to determine the stock values; system dynamics puts stock variables to the forefront. ${ }^{1}$

- They do not ignore soft variables (such as morale or reputation) where these are known to have a causative influence in the system.

Before addressing some of these characteristics in greater detail it is sensible to offer an overview of the adjunct field of systems thinking. This is sometimes described as qualitative system dynamics for its provenance is based upon diagramming or mapping techniques, primarily influence diagrams (ID) or causal loop diagrams (CLD). It was not until the 1970's, nearly fifteen years after the publication of Forrester's early industrial models, before such diagrams started to appear. Their origins can be traced back to Maruyama (1963); Goodman's text (1974) portrays some seminal examples.

\section{SYSTEMS THINKING}

The use of diagramming techniques in the analysis of a system has a long history going back to the block diagrams of control and electrical engineering. However, the qualitative strand associated with system dynamics emphasises the feedback loops present in the system. Feedback is an essential building block of system dynamics whereby information about the current state of the system is used to regulate controls on the resource flows and it underscores the endogenous point of view. For instance, if stocks of manufactured goods are beginning to over-accumulate, it is necessary to either cut back on production throughput or inaugurate a sales drive or both.

These mapping techniques are not mandatory in a system dynamics study. On the other hand there are those who argue such methods, of themselves, have the capacity to generate insight and can help form a consensus for policy change in a problem system. See for instance the testimony from Merrill et al (2013) concerning a health application. They state: "As a tool for strategic thinking on complicated and intense processes, qualitative models can be produced with fewer resources than a full simulation, yet still provide insights that are timely and relevant". Books have appeared which focus exclusively on such mapping techniques, for instance Ballé (1994) and Sherwood (2002), to the exclusion of formal simulation models which are described in section 3 below. Whether such diagrams alone can be considered advantageous in the overall practice of system dynamics has long been the subject of debate in the field. An exchange between Coyle and Homer and Oliva occupied many pages of the System Dynamics Review in 2000-2001. See Coyle (2000; 2001) and Homer and Oliva (2001).

Although the proponents of the need for formal system dynamics models remain implacable, some authors and organisations have prospered in the propagation of systems thinking techniques. Pegasus Communications has for many years published the magazine The Systems Thinker and Peter Senge's reputation as a managerial thought leader was founded on his book The Fifth Discipline (1990) and its associated Fieldbook (1994). It was these sources that, primarily, introduced 'behaviour over time' sketch graphs together with the notion of system archetypes as additional tools in the armoury of systems thinking.

\subsection{Behaviour Over Time Graphs}

Consider the chart in figure 1. It represents some (hypothetical) data for two local authorities showing the percentage of girls who were classed as overweight in 2012.

\footnotetext{
${ }^{1}$ Research has shown that even well-educated people find it difficult to infer the behaviour of a stock variable given known behaviour of the flows affecting that stock.
} 


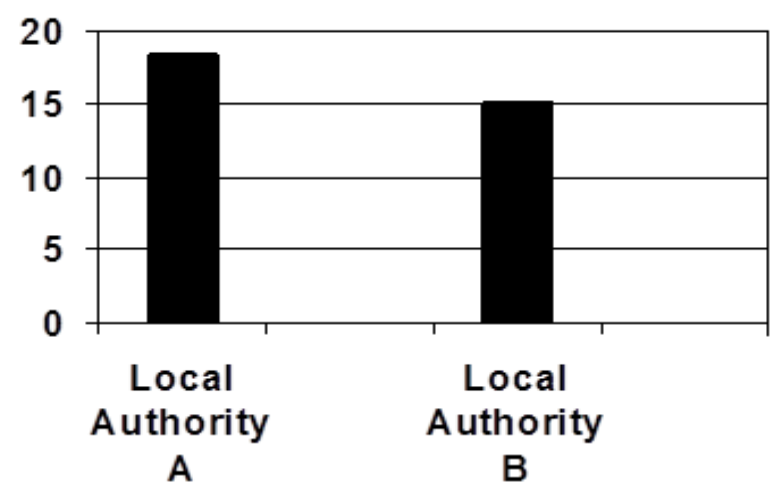

\% Overweight

Figure 1 Prevalence of overweight girls aged 10 - 15 years in two local authorities in 2012

It is a static graph and, as such, conveys limited and what could be misleading information. At first, an examination of the data would appear to suggest that local authority A has a more serious public health situation on its hands than local authority B. However, re-framing the situation using a behaviour over time graph paints an altogether different picture (see figure 2). It is clear that local authority B is more in need of a public health intervention. Consideration of the dynamics in a system is vitally important.

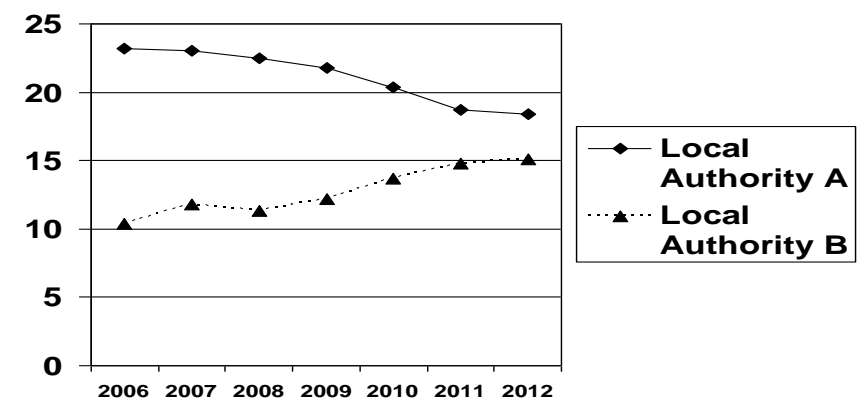

Figure 2 Behaviour over time graph of the prevalence (\%) of overweight girls, 2006-2012

\subsection{Principles of Influence (or Causal Loop) Diagrams}

This section examines the building blocks of the mappings which have come to constitute the heart of systems thinking - the diagrams known as influence diagrams (ID) or causal loop diagrams (CLD). There is no counterpart to an ID or CLD in discrete event simulation. There one progresses to the development of an activity cycle diagram as the initial framework on which the computer simulation model is constructed. That is to say the field of discrete event simulation does not offer an optional diagramming phase which, of itself, is capable of generating insight.

Some practitioners have expressed the view that, in certain instances, an intervention based on systems thinking diagrams is sufficient to unearth the insight necessary to achieve a profound effect on system performance. The argument is bound up with project resources: models as mappings absorb less costs and can still produce insights which are timely and relevant.

A simple example of an influence diagram is given in figure 3. Here we see the basic process underlying a firm's organic growth. As average profits increase they are re-invested to the future benefit of the organisation (positive links). 


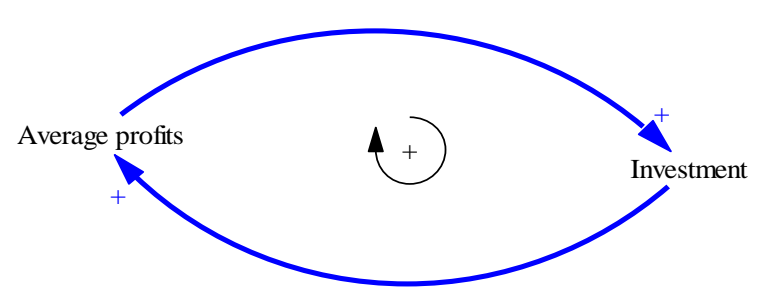

Figure 3 Simple positive feedback loop (Note: the loop descriptor in the middle should flow in the same direction as the loop, in this case clockwise)

Other examples of positive links are: sales per unit time of a durable product increase the customer base; revenues received increase the cash balance; students enrolling on a course increase the total student population.

Note that the + sign not only implies that an increase in one variable causes an increase in another but also, alternatively, that a reduction in one variable causes a reduction in another. In the example in figure 3 a reduction in average profits engenders a reduction in investment. A more obvious example is when rumours of a firm's financial health lead prospective customers to decline to engage with it.

Let us now consider a negative loop. The underlying influence created by such a loop is one of a controller. If movement occurs in the dynamics in one direction then a countervailing force pushes against that momentum to establish the original (or a new) equilibrium. The entire discipline of control engineering is concerned with how negative loops can be represented as physical controllers in machinery of all types, for example the auto-pilot in modern aircraft and the thermostat in a heating system.

Figure 4 shows an example of a simple negative loop taken from the domain of stock control. The very word 'control' reflects the nature of what is going on. As stock levels increase then replenishment ordering is cut, or vice versa (negative link). The change in the flow of orders directly affects the stock level and thus completes the loop. Other examples of negative links are: a perceived reduction in the numbers of a particular workforce will lead to an increase in employee recruitment; an increase in spending on wages will lead to a fall in an organisation's cash balances.

In selecting the sign to place on a given arrowhead (establishing link polarity) ${ }^{2}$ it is important not to take into account other influences that may be simultaneously operating. The Latin maxim of ceteris paribus, so common in elementary economics texts, needs to be adhered to: i.e. let other factors remain constant. Therefore, the only consideration in assigning link polarity is: what effect will a change in the variable at the tail of the arrow have on the variable at its head?

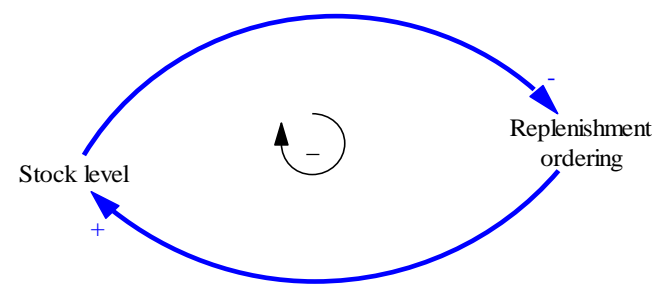

Figure 4 Simple negative feedback loop

Two mutually connected negative relationships create a positive loop. Consider figure 5 where an increase in staff turnover (in a close working team) will lead to a fall in morale which in turn will lead to a further increase in staff turnover.

\footnotetext{
${ }^{2}$ In recent years some authors have replaced the use of + and - by s (same) and o (opposite).
} 


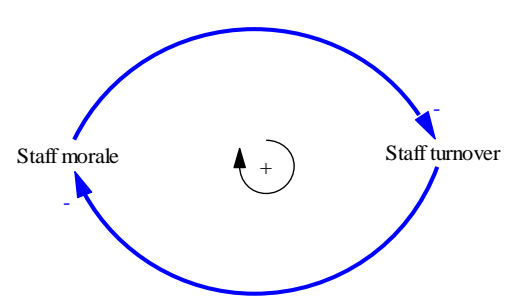

Figure 5 Two mutually causative negative relationships create a positive loop

In determining the loop (as opposed to link) polarity there are two methods available. One can enter the loop at any given point, and start with, say, an increase in that variable and trace around the effect. If one returns to that point with a further increase then the loop is positive, but if the initial increase has resulted in a decrease then the loop is negative. An arguably easier approach is to add up the number of negative links in the loop: if the number is zero or is even, then the loop is positive and if it is an odd number then the loop is negative. The loop polarity is the algebraic product of the number of negative signs, e.g. three negatives multiplied together yield a negative result, hence a negative loop.

\subsection{From Diagrams to Behaviour}

The determination of loop polarity is not merely an exercise for its own benefit but rather serves as a precursor to being able to infer the behaviour mode of the loop if it were to be 'brought to life'. Loop dynamics differ between negative and positive loops so it is essential to determine loop polarity. A positive loop produces dynamics which reinforce an initial change from an equilibrium point and so underpin growth and decay behaviour patterns. A pure positive loop in growth mode will produce exponentially increasing behaviour. A negative loop, on the other hand, will generate equilibrating behaviour such that any shift away from an initial equilibrium point will produce a compensating force driving it back towards that point (or indeed a new equilibrium). Introducing a delay into a negative loop will induce an oscillation in the behaviour. It is this knowledge which can aid in model conceptualisation when time-series data is available. After smoothing out any noise which may be present, an oscillatory behaviour pattern is indicative of a system dominated by a negative loop or loops; one which exhibits growth or decay would suggest that a positive loop is at work somewhere. An oscillatory behaviour associated with a trend up or down would suggest the need for a model conceptualisation based around a combination of negative and positive loops.

In order to further develop this idea of behaviour generated by different feedback loops it is necessary to move away from the single loop examples above to a more realistic real-world situation where multiple loops are at play. For instance, the example at figure 6 portrays a simple product diffusion model where initial sales generate further growth through 'word-of-mouth' effects but this growth is ultimately curtailed by market limitations of one form or another. Because this system structure also underpins the dynamics of an epidemic in a closed population (e.g. passengers on a cruise liner) the variables named for the diffusion example have been duplicated by the equivalent epidemic variables: the same system structure can underpin quite widely different situations!

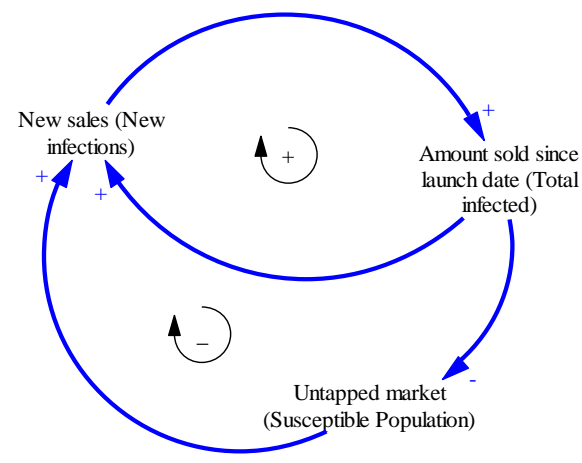

Figure 6 Influence diagram showing two loops and two different examples: diffusion dynamics and epidemics underpinned by the same system structure 


\section{Dangerfield}

Also brought out by figure 6 is the associated concept of loop dominance. As the structure plays out over time, the positive loop is dominant initially - that is to say it has the control of system behaviour in the early stages while the word-of-mouth effects are at play. Ultimately the market limits begin to take over. There are fewer and fewer people who do not have this product and so the capability of making further new sales is diminishing by each passing week. Now the negative loop assumes dominance in system behaviour and growth slows. Figure 7 shows the resultant behaviour: s-shaped growth where the transition from growth to market maturity coincides with the switch in loop dominance. Technically this is at the point of inflection on the cumulative curve, a point where the sales per unit time (not shown) reach a peak and start to fall.

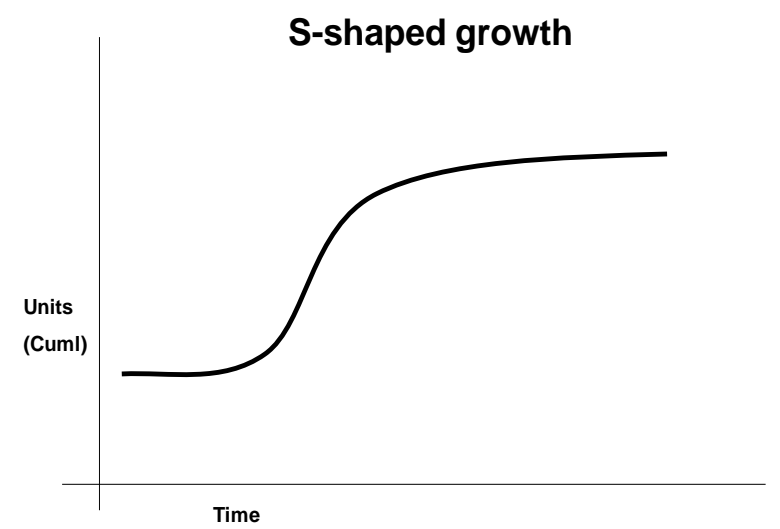

Figure 7 S-shaped (or sigmoidal) growth generated by coupled positive and negative loops

\section{SYSTEM DYNAMICS}

It is now appropriate to move forward and to consider the conceptualisation and formulation of a formal system dynamics simulation model. As mentioned earlier there is no essential requirement to preface the creation of an SD model with an influence diagram. There are those who argue that an influence diagram can aid in the definition of system content (and model boundary) but there is no direct linkage between such a diagram and the formal simulation model. This is in contrast to the stock-flow diagram: here the stocks (levels) and flows (rates) need to be explicitly present in the equation listing for the model.

\subsection{Principles of Stock-flow Diagramming}

The stock-flow diagram in system dynamics is the counterpart to the activity cycle diagram (ACD) in discrete event simulation. Although the flows may not result in a cycling of resources as such (which is common in DES), each diagram is there to underpin the formal model and the quantitative expressions which define its constituent elements.

System dynamics flow rates are depicted by a tap-like symbol which indicates a device which can control the flow, equivalent to policy controls in the real world. A stock is represented by a rectangle and here there exists an unfortunate misalignment in the DES and SD diagramming conventions. In DES a rectangle is reserved for an activity - an active state. A stock in SD is a 'dead' state, equivalent to a queue in DES. Figure 8 is an example of what might be part of the stock-flow diagram underpinning an SD model of a nation's education system.

It is important to note that the boundary of the flow at each edge of the system is represented by a cloud-like symbol. Consideration of the resource beyond these points is outside the scope of the model. Also, the stocks and flows must alternate along the sequence. The incoming flow adds to a stock while an outgoing one drains it. Only one resource can be considered along any process flow. So, for example, what starts as a flow of material (or product) cannot suddenly be transformed into a flow of finance. Thus, separate flow lines need to be formulated for the various different resources being considered in the model. 'Resource' can be taken to be a product class, financial flow, human resources, orders, capital equipment and so on. Clearly the more resource flows being considered the more complex the model and the more equations it will comprise. 


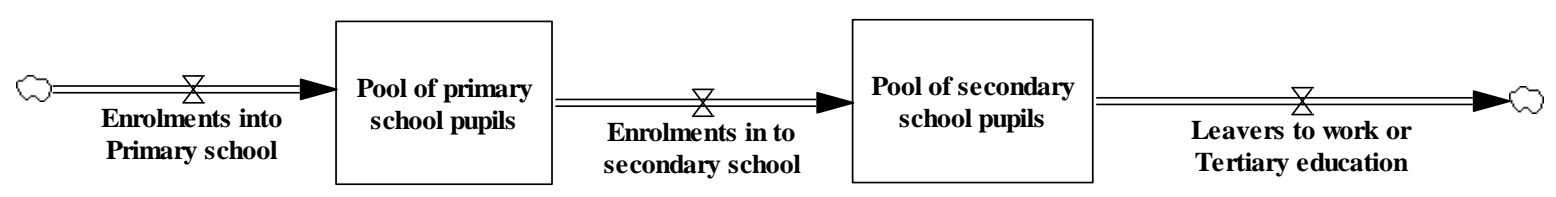

Figure 8 Example of a single flow process in a stock-flow diagram

Additional arrangements are possible. One can have an inflow to a stock without an outflow (or vice versa). A flow structure may call for more than one inflow and/or outflow. In certain cases the flow might actually form a cycle. This can happen, for instance, if one is modelling a manufacturing recycling process often described as 'reverse logistics' or a 'closed loop supply chain'. Although such flow arrangements do constitute a loop or cycle they are in no circumstances a feedback loop. As will be described later, a feedback loop is based on information feedback.

\subsection{Model Purpose and Model Conceptualisation}

Getting started can be the greatest difficulty in the creation of a useful SD model. One starts with the proverbial blank sheet of paper. Experience over many years has taught the author that two fundamental aspects of SD model conceptualisation are: firstly, being able to write in one sentence the purpose of the model and, secondly, ensuring the stock-flow representation is 'right'. This latter term is deliberately placed within inverted commas because no model can ever be perfectly correct and represent the ultimate truth, but it is meant to suggest that a great deal of thought needs to go into deciding which resource flows to include, and how to structure those flows as bald stocks and flows with no consideration of any other variables or constants at this juncture - these can be usefully termed the spinal stock-flow structures (see example in figure 8). Where a client is involved they need to 'buy into' that raw stock-flow diagram and the written definition of model purpose before any further model formulation work is undertaken. Several iterations of this first conceptualisation are typically necessary. The above advice also underlines the point made earlier about influence diagrams - they are not always necessary as a precursor to formal model creation. For this task the stock-flow diagram reigns supreme.

A particularly useful precept, first expressed by Forrester in Industrial Dynamics (1961), is to define the level (stock) variables. These would still be visible if the system metaphorically stopped (e.g. employees in a factory; cash in the firm's bank accounts). Next, consider what might be flowing into and/or out of those stocks. These flows would, of course, not be visible if the system 'stopped'. All the time it is necessary to remember that a number of different spinal stock-flow modules may be required in order to fully conceptualise the model in line with the agreed model purpose.

\subsection{Adding Auxiliaries, Parameters and Information Links to the Spinal Stock-flow Structure}

In order to flesh out the spinal stock-flow structure it is necessary to embellish it with other explanatory variables (called auxiliaries), together with parameters. In general one follows the oft-restated mantra: rates (flows) affect levels (stocks) via resource flows, while levels (stocks) affect rates via information (feedback) links. The sequence is:

$\begin{array}{ll}\text { Resource flows } & >\text { System state } \\ \text { System state } & >>\text { Information to management } \\ \text { Information to management } & >\text { Managerial action } \\ \text { Managerial action } & >>\text { Resource flows }\end{array}$

This is the essential expansion of the concept of the feedback loop which is illustrated in figure 9. 


\section{Dangerfield}

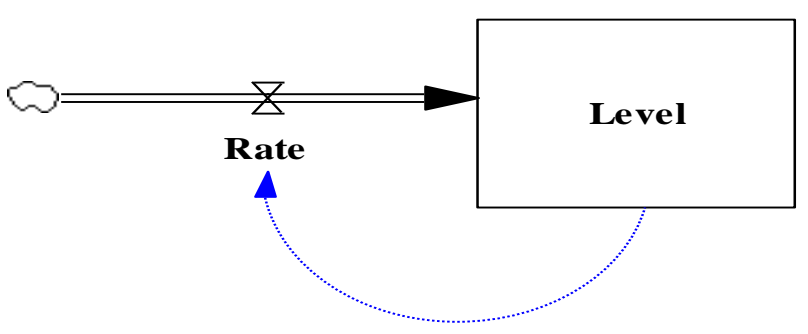

Figure 9 A simple feedback loop in stock-flow symbolism

In general more complexity will be required and other variables, which are neither stocks nor flows, of necessity have to be introduced - these are termed auxiliaries. These reflect variables which, in a business model, lie in the managerial planning and information system. Thus, any variable which is intended to represent something planned, desired, a target, or a management goal would be modelled by an auxiliary variable. Consider the augmented stock-flow diagram in figure 10. Here the concept of a desired workforce has been added to explain the recruitment rate on the spinal flow. It would seem intuitive that recruitment policy might be explained by a comparison between the desired workforce and what one currently possessed.

The level of sophistication can increase, however. To jump ahead a little there is another item which would need to be added, namely the adjustment time for eliminating any discrepancy between the desired and actual workforce. Workforce adjustment time would be a parameter and would mimic the average time to advertise and recruit new people or to give notice of redundancy and fire them if business conditions dictated it. Moreover, it might be necessary to have two different parameter values if the average time constant were thought to be different for recruitment and firing processes. Additionally, there may be a need to introduce other auxiliary variables in order to better define the desired number of employees. In fact, chains of auxiliaries are often created in order to effect a proper definition for the flow variable. System dynamics models tend to reflect real-world causes and effects very closely and this is one of the reasons why it is such a powerful methodology and why the total number of variables and parameters can rapidly escalate over and above the original number of variables on the spinal flows.

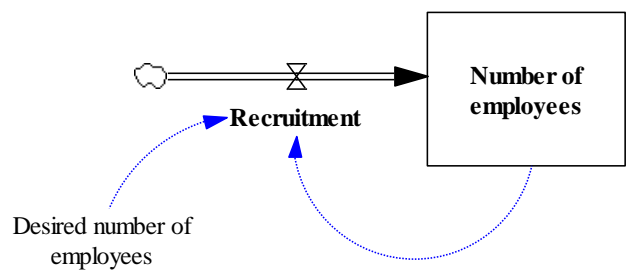

Figure 10 Auxiliary variable and information links added to the spinal flow

\subsection{Equation Writing and Dimensional Checking}

Undoubtedly for many the most challenging task in SD model formulation is the composition of the equations for the rates and auxiliary variables. In modern SD software the stock variables are automatically created because the system can 'see' what is flowing into and/or out of a stock. These integration equations take the form:

Stock value at current time $\mathrm{t}=$ old value of stock at $\mathrm{t}-\mathrm{dt}+\mathrm{dt} *$ (Inflows - Outflows) [dt is 'delta time' an infinitesimally small slice of time in mathematical calculus; approximated by a fraction of the time unit on a computer.]

System dynamics simulations exhibit a constant time advance (unlike DES) and through this process the equations describing the flow rates (which are, in effect, differential equations) are converted to difference equations and solved to yield the values of the stocks as in the example above. In the earlier SD literature the time increment was termed dt to reflect the 'with respect to' element commonly seen in differential calculus; TIME STEP is often employed nowadays. Its value is normally restricted to a binary fraction $\left(1 / 2^{\mathrm{n}}\right.$, for $\left.\mathrm{n}=0,1,2 \ldots\right)$ because of the way computers handle real numbers; 


\section{Dangerfield}

by this means the greatest accuracy is achieved in determining the value for the system (reserved) variable Time. Clearly at the beginning of the simulation an 'old' value is needed to initialise the stock and this is termed an initial value. All stocks must have an associated initial value declared in order for the time advance process of the simulation to get started.

However, whilst formulation of the integration equations can be left to the software, this is not the case with rate and auxiliary equations. Here the user needs to compose the expression based upon the known informational influences evident in the developing stock-flow diagram. To this end it is recommended that the influence links are entered on the diagram before building the equation.

Structuring the equation is unavoidably bound up with units (or dimensional) checking. Most with a background in the physical sciences and engineering will know that any equation describing a realworld process needs to have the units balanced on each side of the ' $=$ ' sign. Thus, if the units on the left are $\$ / y r$ then the expression on the right side needs to algebraically decompose to $\$ / y r$. Further, if any terms on the right side are added or subtracted then each individual term needs to have the same units as the variable on the left side.

In the integration equation above, the ' $\mathrm{dt}^{*}$ ' element on the right side is necessary in order for the units to balance since the flows will be in terms of units/time. The dt term is a time interval and so we have time*units/time $=$ units and the entire expression is units $=$ units + units - units.

For the formulation of rate and auxiliary equations the user needs to think in terms of the units involved. If the variable concerned is expressed in terms of units/mths then the expression on the right side needs to also be units/mths. Thinking along these lines can actually aid in the formulation of the expression. You should know what units the rate or auxiliary is measured in; the right side needs to duly conform.

Let's consider some simple examples:

(1) The Accounts Payment Rate (APR) is known to be influenced by the value of Accounts Payable (AP) and a Delay in Making Payment (DMP).

$\mathrm{APR}=\mathrm{AP} / \mathrm{DMP}$ and $\$ / \mathrm{mths}=\$ / \mathrm{mths} \quad[$ This describes a flow of funds used to settle accounts.]

(2) The annual Out-Migration Rate (OMR) from a certain region of a country is dependent on the Population (POP), the normal Fraction of People Leaving (FPL) and the Departure Migration Multiplier (DMM).

The multiplier term could be there to account for periods of time when the normal fraction departing is tweaked as a result of, say, a temporary incentive. Where such constructs are employed in SD models they are inevitably dimensionless, that is to say they have no units. As well as a multiplier, any fraction, proportion, percentage or an index number would be dimensionless and be given units of ' 1 '.

So we have: $\mathrm{OMR}=\mathrm{POP} * \mathrm{FPL} * \mathrm{DMM}$ and persons/yrs= persons $* 1 / \mathrm{yrs} * 1$

Why is the FPL term in units of $1 / \mathrm{yrs}$ ? This is because it is the number of persons leaving each year divided by the number there to start with, or (persons/yrs)/persons $=1 / \mathrm{yrs}$. The same idea applies with an interest rate which is $(\$ / \mathrm{yrs}) / \$=1 / \mathrm{yrs}$ (i.e. a percentage, which is dimensionless, but which can change over time).

Below are listed two possible equations to describe the Production Rate (PR). It is interesting to note that each is quite different but both are dimensionally balanced.

(3) Production Rate (PR) is a function of the Workforce (WF) and their Productivity (PROD). Productivity can crop up in a lot of business models and its dimensions can cause difficulty. It is a compound dimension expressed as (output) units/person/time unit, or (units/(persons*time)). 
So we have: $\mathrm{PR}=\mathrm{WF} * \mathrm{PROD}$ and units/time $=$ persons $*($ units $/($ persons $*$ time $))$

(4) Production Rate (PR) is related to the Average Sales Rate (ASR), together with a Correction for a Stock Discrepancy (CSD) and a Correction for a Backlog Discrepancy (CBD). The correction terms will be accounted for separately in the model and they describe the product units produced per time unit that will eliminate any discrepancy between what is desirable and the state of affairs that exists.

So we have: $\mathrm{PR}=\mathrm{ASR}+\mathrm{CSD}+\mathrm{CBD}$ and units/time $=$ units/time + units/time + units/time

Which formulation for PR is the correct one? Either could be and there may indeed be other formulations which occur to the reader. The formulation employed is the one which is most appropriate given the purpose of the model and the circumstances prevalent in the actual system being modelled. A useful categorisation of commonly found formulations for rate and auxiliary equations is set out in the classic SD text by Richardson and Pugh (1981) and also in Sterman (2000). In addition the aspiring modeller should also study the many model listings provided by SD experts in texts and as supplementary material in journal articles.

To conclude a more complicated equation formulation example is described. It concerns the need to formulate an expression for the Extra Labour (EL) required to eliminate a greater than normal backlog of orders. Many operations experience this challenge, especially if there are seasonally induced gluts in orders. It is not feasible to employ a large workforce throughout time and it falls to the management to recruit more people when a very high backlog situation arises.

An initial formulation might be:

$$
E L=(O B-N O B) / P T A B
$$

where $\mathrm{OB}=$ Order Backlog

$\mathrm{NOB}=$ Normal Order Backlog

$\mathrm{PTAB}=$ Planned Time to Adjust the Backlog

EL is obviously dimensioned as 'persons' and the expression is:

$$
\text { persons }=\text { units/time }- \text { units/time }
$$

The equation is not balanced dimensionally. It is necessary to introduce another variable (or constant) which will relate units/time to persons. A moment's thought should make one realise that the concept of worker productivity (see example 3 above) is missing and so an additional parameter is required, say Normal Productivity of Labour (NPL). After some further thought it will be established that this parameter needs to be included in the denominator of the expression and as a multiplier. We now have:

$$
E L=(O B-N O B) /(P T A B * N P L)
$$

Extracting just one of the terms in the numerator for the dimensional check yields:

$$
\text { persons }=\text { units } /(\text { time } *(\text { units/ } / \text { persons } * \text { time })))
$$

and the two 'time' and 'units' elements cancel, leaving persons= persons and the equation is shown to be dimensionally correct. In the final step above it might be necessary to recall the mathematical dictum often chanted in school: "Invert the divisor and multiply".

\section{A COMPLETE SYSTEM DYNAMICS MODEL}

The following gives a description of a problem at an IT Service Centre. We will conceptualise a model to address this issue and then develop and run simulation experiments (using Vensim) to explore a changed workforce recruitment policy which can improve dynamic performance. For more on workforce modelling see Cave \& Willis (2020). 


\section{Dangerfield}

An IT service centre is staffed by 25 skilled specialists who respond to problems and difficulties which IT users make them aware of. When a user raises an issue a ticket is created containing a unique reference number for that problem. Once the matter is dealt with the ticket is completed and so is removed from the list of the IT ticket caseload.

From a historical perspective there is a normal turnover of IT staff. HR data shows around 3 staff have departed per month $(12 \%)$ and the management have traditionally recruited new staff at the same average rate of departing staff. Typically, there will be 200 outstanding tickets in the caseload and these are raised at the rate of 500 per month and indeed completed at the same rate. So, an IT specialist will, on average, complete 20 tickets per month.

Over recent months there has been a surge in workload arising from a new software installation which users are having trouble familiarising themselves with. This has put pressure on the IT staff who are having to increase their workload (working longer hours etc) dealing with the surge in ticket submissions. In fact, it is having repercussions in terms of team morale and a lot more IT specialists are leaving the company than has hitherto been the case. The management are of course recruiting new skilled IT specialists but it doesn't seem to be adequately addressing the problem and the IT workload issue is not improving.

To conceptualise this issue we need to consider two resources: people and orders (tickets).
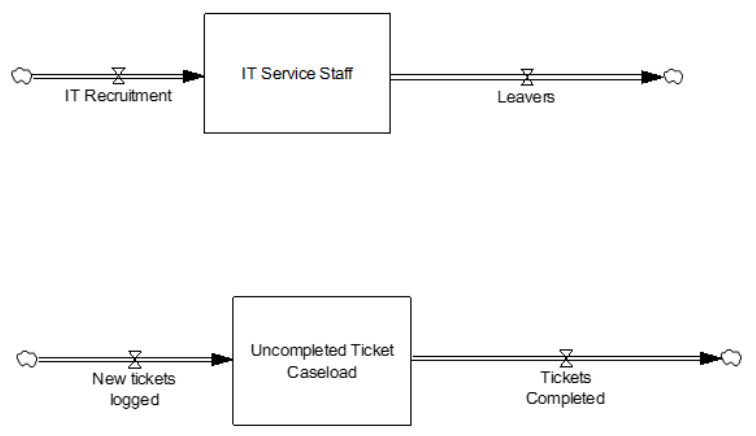

Figure 11 Initial raw stock-flow conceptualisation of the workload problem

We create two spinal stock and flow resource modules (as shown in figure 11). Next, we need to write expressions to capture the informational links which drive the flows. These will be either natural forces or managerially imposed forces (i.e. policies). In so doing we will need to introduce additional variables which lie in the information systems driving the flows; these are called auxiliary variables. This leads to figure 12. Also shown are the detailed equations and parameter values as produced by the Vensim model documentation feature.

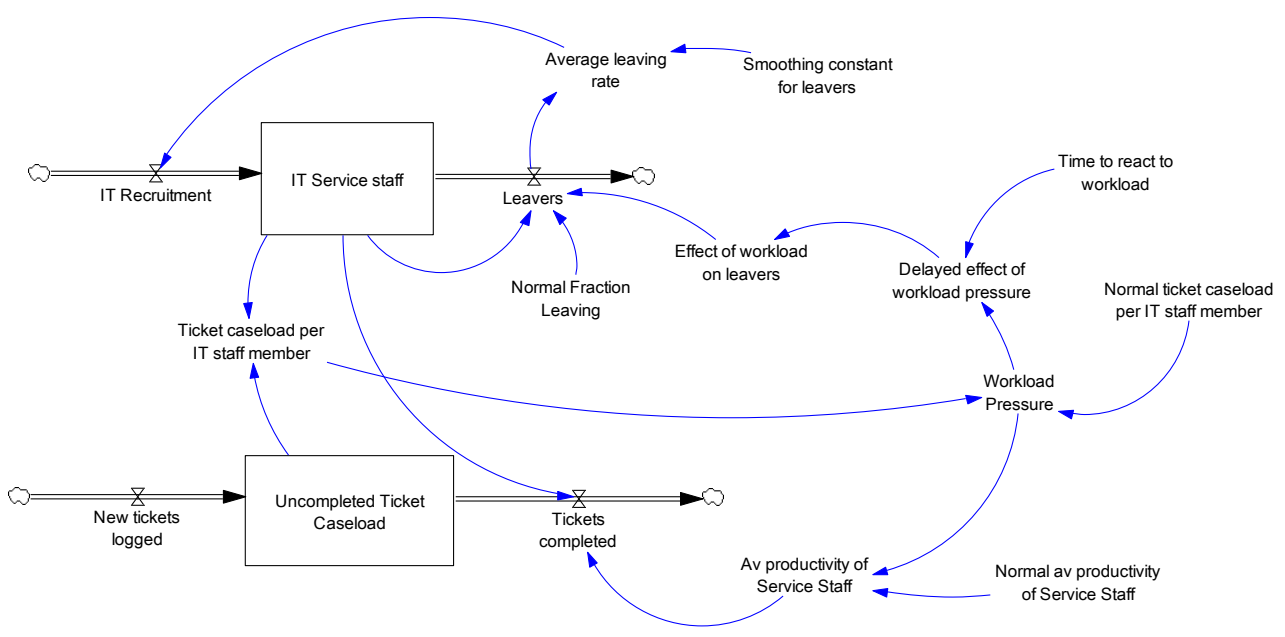

Figure 12 Model with information links and parameters added 
(01) Av productivity of Service Staff=Normal av productivity of Service Staff*Workload Pressure Units: tickets/(persons*mths)

(02)Average leaving rate=SMOOTH(Leavers, Smoothing constant for leavers )

Units: person/mths

(03)Delayed effect of workload pressure=SMOOTH3(Workload Pressure, Time to react to workload )

Units: Dmnl (dimensionless)

(04)Effect of workload on leavers= WITH LOOKUP (Delayed effect of workload pressure, ([(1,0)-(2,8)],(1,1),(1.07951,1.12281),(1.19266,1.33333),(1.33639,1.78947),

$(1.48318,2.14035),(1.58104,2.52632),(1.71254,2.98246),(1.82875,3.85965)$,

$(1.88073,4.31579),(1.93578,4.80702),(2,5.64912)))$

Units: Dmnl (dimensionless) This makes use of a Vensim X-Y lookup as shown below.

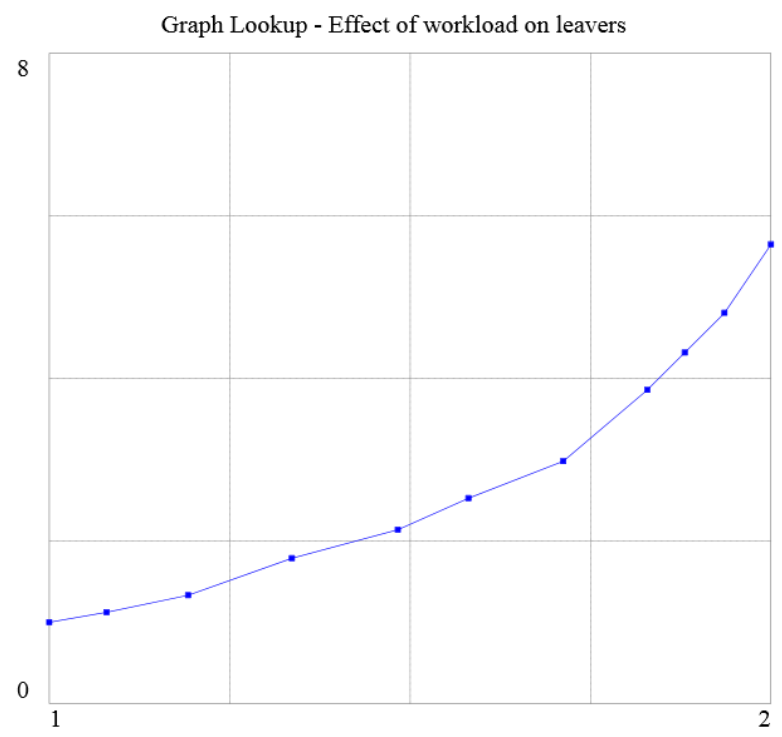

(05)FINAL TIME $=24 \quad$ Units: mths (The time horizon for the simulation.)

(06)INITIAL TIME $=0 \quad$ Units: $m$ ths (The initial time for the simulation.)

(07)IT Recruitment=Average leaving rate Units: persons/mths

(08)IT Service staff= INTEG (-Leavers+IT Recruitment, 25) Units:persons

(Equation provided by Vensim - apart from initial value of 25)

(09)Leavers=IT Service staff*Normal Fraction Leaving*Effect of workload on leavers Units: persons/mths

(10)New tickets logged $=500+\operatorname{STEP}(125,6)$

Units: tickets/mths (Mimics the increase in workload: sudden 25\% increase at $\mathrm{t}=6$ )

(11)Normal av productivity of Service $\mathrm{Staff}=20$

Units: tickets/(persons*mths)

(12) Normal Fraction Leaving $=0.12$

Units: $1 / \mathrm{mths}$

(13)Normal ticket caseload per IT staff member $=8$

Units: tickets/person

(14)SAVEPER = TIME STEP

Units: mths $[0,24]$

The frequency with which output is stored.

(15) Smoothing constant for leavers $=3$

Units: mths

(16)Ticket caseload per IT staff member=Uncompleted Ticket Caseload/IT Service staff 


\section{Dangerfield}

Units: tickets/person

(17)Tickets completed=IT Service staff*Av productivity of Service Staff

Units: tickets/mths

(18)TIME STEP $=0.03125$

Units: mths [0,24]

The time step for the simulation.

(19) Time to react to workload $=6$

Units: mths

(20)Uncompleted Ticket Caseload= INTEG (New tickets logged-Tickets completed, 200)

Units: tickets (Again, provided by Vensim.)

(21)Workload Pressure=Ticket caseload per IT staff member/Normal ticket caseload per IT staff member

Units: Dmnl (dimensionless)

From this base case model we can show that the recruitment policy for new staff is too reactive. Figure 13 paints the poor performance.
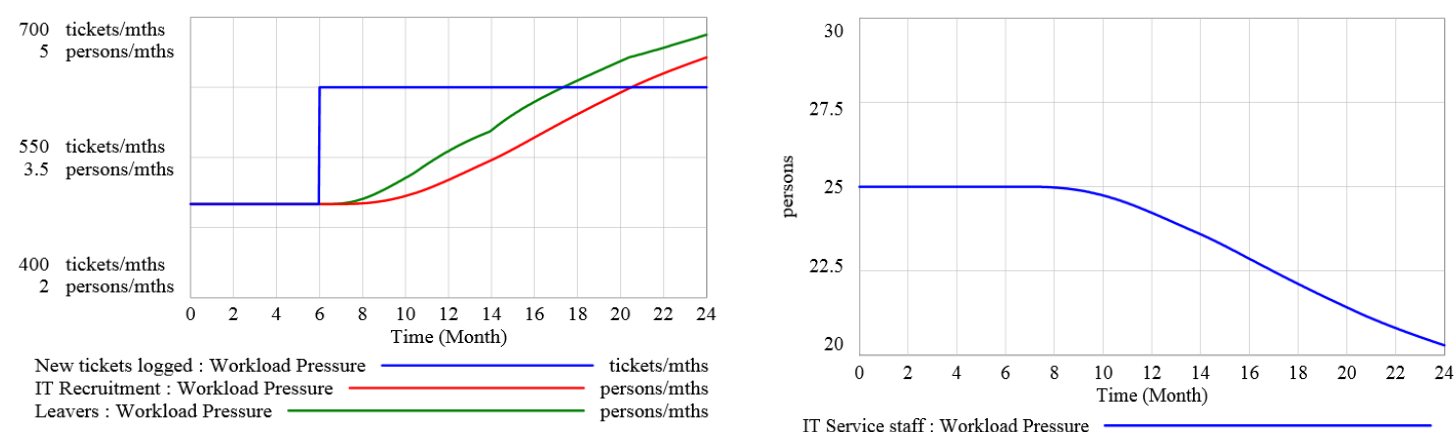

Figure 13 Output graphs from the base case

We need a policy based upon an improved 'early warning'. A possibility would be to monitor new tickets logged and then introduce the concept of desired IT service staff numbers based upon this. This revised policy is depicted in the model shown in figure 14.

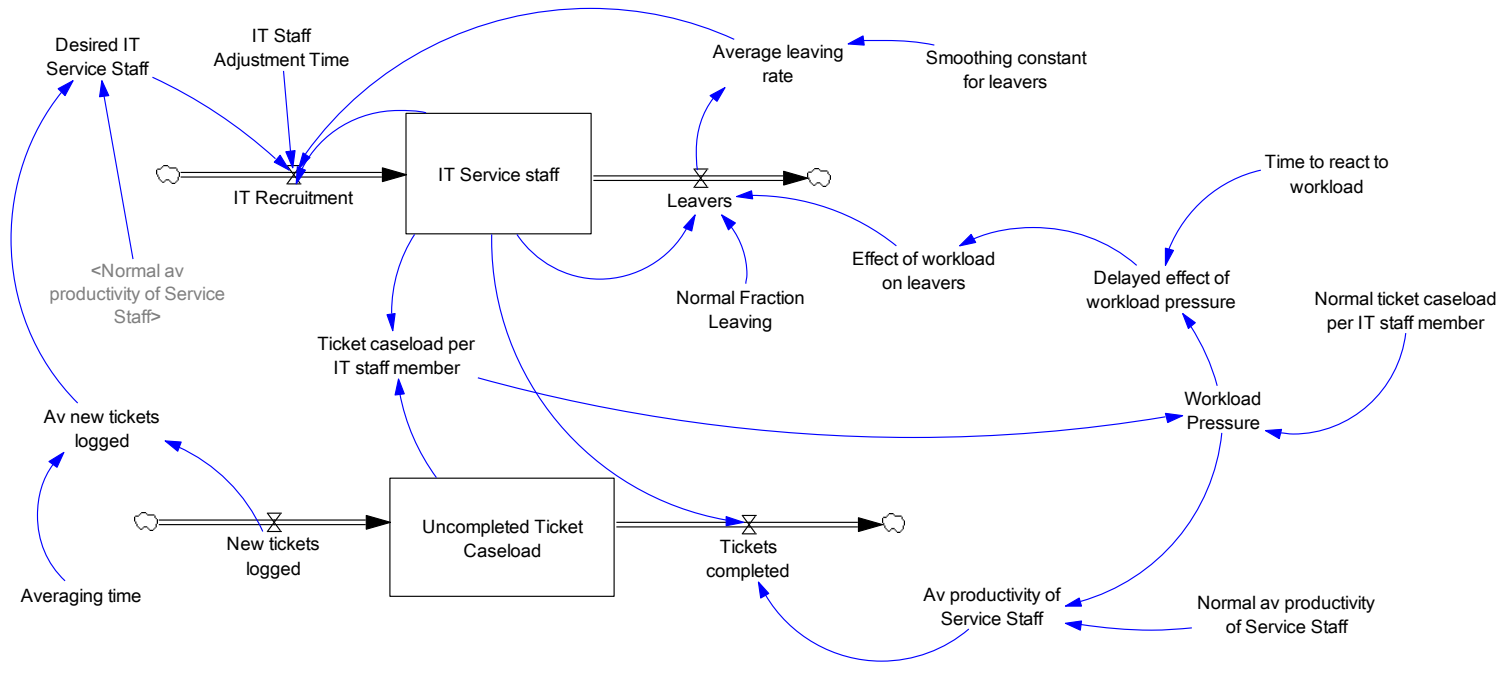

Figure 14 Revised recruitment policy for IT staff 


\section{Dangerfield}

The revised / additional equations are listed below:

(01)Av new tickets logged=SMOOTH(New tickets logged, Averaging time)

Units: tickets/mths

(02)Averaging time $=1$

Units: mths

(03)Desired IT Service Staff=Av new tickets logged/Normal av productivity of Service Staff Units: persons

(04)IT Recruitment=(Desired IT Service Staff-IT Service staff)/IT Staff Adjustment Time+Average leaving rate

Units: persons/mths

(05)IT Staff Adjustment Time=1

Units: mths

The new recruitment policy has beneficial dynamic effects shown in figure 15 .
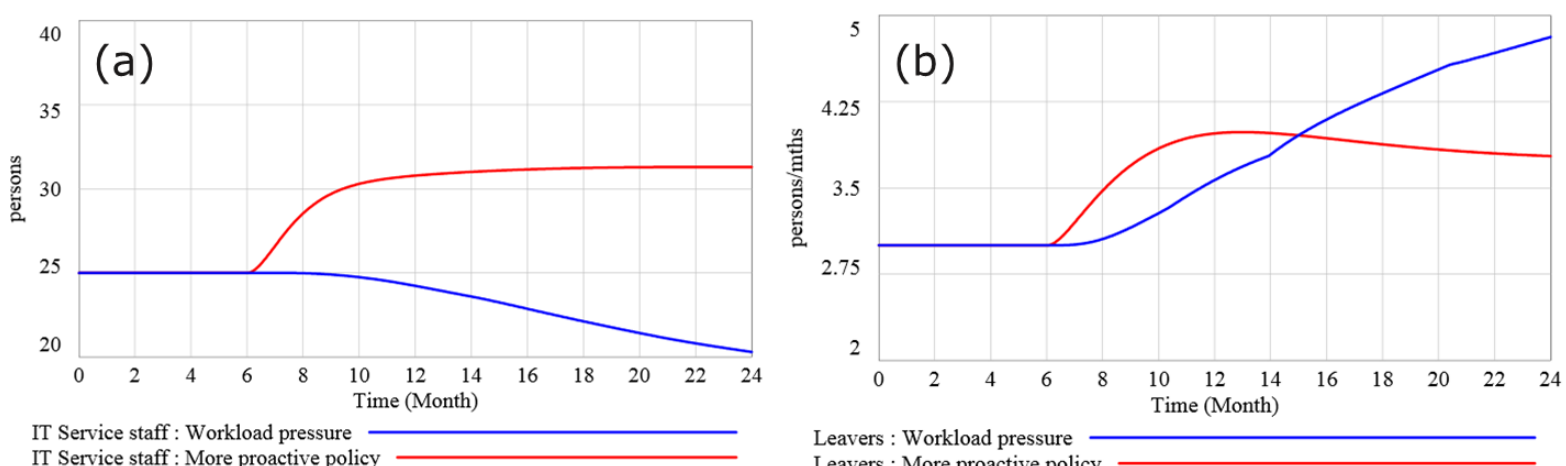

Figure 15 Comparison plots of (a) IT services staff and (b) leavers under existing and revised policies

\section{FURTHER READING}

It is impossible in this overview paper to fully do justice to what is now a significant methodology in the socio-economic, managerial, health, biological, environmental, energy and military sciences. However, three contemporary books will take the interested reader much further. These are purely the author's choice and they are listed in order of page count.

John Sterman's book (982pp) has arguably the most comprehensive coverage; see Sterman (2000) in the reference list. John Morecroft's (466pp) Strategic Modelling and Business Dynamics: a feedback systems approach, Wiley (2007) offers a very wide coverage of systems thinking and system dynamics and incorporates many practical model examples. Thirdly, Kambiz Maani and Bob Cavana have written a second edition of their offering (288pp): KE Maani and RY Cavana, (2007) Systems Thinking, System Dynamics: managing change and complexity, Pearson Education NZ (Prentice Hall), Auckland.

All these books come with a CD-ROM and/or a website which provides specimen models to be run and allows scenario experiments to be conducted. Exercises and instructor's manuals are also available.

An expanded version of the above material can be found in chapter 3 of the book by Brailsford, Churilov and Dangerfield (2014). This book covers both discrete-event simulation and system dynamics.

The book edited by Dangerfield (2020;540pp) attempts to portray both methodological aspects and contemporary applications of SD now the field has just passed its $60^{\text {th }}$ anniversary.

\section{REFERENCES AND SELECT BIBLIOGRAPHY}

Ballé M (1994) Managing with Systems Thinking, McGraw Hill, London.

Barlas Y (1996) Formal Aspects of Model Validity and Validation in System Dynamics. System

Dynamics Review, 12:3, 183-210. 


\section{Dangerfield}

Brailsford S, Churilov L and Dangerfield B (Eds ) (2014) Discrete Event Simulation and System Dynamics for Management Decision Making, Wiley, Chichester. See chapters by Robinson; Elder (DES \& SIMUL8) and by Dangerfield; Jones (SD \& VENSIM).

Cave S and Willis GJ (2020) System Dynamics and Workforce Planning. In: System Dynamics: Theory and Applications, Dangerfield BC (Ed.), Springer, New York. 431-457.

Coyle RG (2000) Qualitative and quantitative modelling in system dynamics: some research questions, System Dynamics Review, 16:3 225-244.

Coyle RG (2001) Rejoinder to Homer and Oliva, System Dynamics Review, 17:4 357-363.

Coyle G and Exelby D (2000) The Validation of Commercial System Dynamics Models, System Dynamics Review, 16:1, 27-41.

Dangerfield BC and Roberts CA (1996) An Overview of Strategy and Tactics in System Dynamics Optimisation, Journal of the Operational Research Society, 47:3 405-423.

Dangerfield BC and Roberts CA (1999) Optimisation as a statistical estimation tool: an example in estimating the AIDS treatment-free incubation period distribution, System Dynamics Review, 15:3, 273-291.

Dangerfield BC (2009) Optimization of System Dynamics Models. In: Meyers RA (ed.) Encyclopaedia of Complexity and Systems Science, Springer, New York 9034-9043. Reprinted in Complex Systems in Finance and Econometrics, Springer, New York, 2011, 802-811.

Dangerfield BC (Ed.) (2020) System Dynamics: Theory and Applications, Springer, New York.

Dangerfield BC \& Duggan J (2020) Optimization of System Dynamics Models, In: System Dynamics: Theory and Applications, Dangerfield BC (Ed.), Springer, New York, 139-152.

Forrester JW (1958) Industrial Dynamics - a major breakthrough for decision makers, Harvard Business Review, 36:4, 37-66.

Forrester JW (1961) Industrial Dynamics, MIT Press, Mass (Now available from the System Dynamics Society, Albany NY.)

Forrester JW (2007) System Dynamics - a personal view of the first fifty years, System Dynamics Review, 23:(2-3) 345-358.

Forrester JW and Senge PM (1980) Tests for Building Confidence in System Dynamics Models. In: Legasto, AA Jr., Forrester, JW and Lyneis JM (Eds.): System Dynamics. Amsterdam: NorthHolland Publishing Company, 209-228.

Goodman MR (1974) Study Notes in System Dynamics, Wright-Allen Press, Cambridge, MA (Now available from the System Dynamics Society, Albany NY.)

Graham AK and Ariza CA (2003) Dynamic, hard and strategic questions: using optimisation to answer a marketing resource allocation question, System Dynamics Review, 19:1, 27-46.

Hardin G (1968) The Tragedy of the Commons, Science 162:3859, 1243-1248. DOI: 10.1126/science.162.3859.1243

Homer J and Oliva R (2001) Maps and models in system dynamics: a response to Coyle, System Dynamics Review, 17:4 347-355.

Keloharju R and Wolstenholme EF (1988) The Basic Concepts of System Dynamics Optimisation, Systems Practice, 1: 65-86.

Keloharju R and Wolstenholme EF (1989) A Case Study in System Dynamics Optimisation, Journal of the Operational Research Society, 40:3, 221-230.

Maruyama M (1963) The Second Cybernetics: Deviation-Amplifying Mutual Causal Processes American Scientist, 51:2, 164-179.

Meadows DM and Robinson JM (1985) The Electronic Oracle, Wiley: Chichester. (Now available from the System Dynamics Society, Albany NY.)

Meadows DL and Sterman J (2012) Fishbanks: A Renewable Resource Management Simulation https://mitsloan.mit.edu/LearningEdge/simulations/fishbanks/Pages/fish-banks.aspx [Accessed 30 May, 2013]

Merrill JA, Deegan M, Wilson RV, Kaushal, R, Fredericks, K. (2013) A system dynamics evaluation model: implementation of health information exchange for public health reporting. Journal of the American Medical Informatics Association, 20:e131-e138 DOI:10.1136/amiajnl-2012-001289.

Richardson GP and Pugh AL (1981) Introduction to System Dynamics Modelling with DYNAMO, MIT Press, Cambridge, MA. (Now available from the System Dynamics Society, Albany NY.) 


\section{Dangerfield}

Richardson GP (2011) Reflections on the foundations of system dynamics, System Dynamics Review, 27:3 219-243.

Schwaninger M and Grösser S (2020) System Dynamics Modelling: Validation for Quality Assurance. In: System Dynamics: Theory and Applications, Dangerfield BC (Ed.), Springer, New York, 119-138.

Senge P (1990) The Fifth Discipline, Currency Doubleday, New York.

Senge P, A Kleiner, C Roberts, R Ross, B Smith (1994) The Fifth Discipline Fieldbook, Currency Doubleday, New York.

Sherwood D (2002) Seeing the Forest for the Trees: A Manager's Guide to Applying Systems Thinking, Nicholas Brealey, London.

Sterman JD (2000) Business Dynamics: Systems Thinking and Modeling for a Complex World, Irwin McGraw-Hill, New York.

Sterman JD (1984) Appropriate Summary Statistics for Evaluating the Historical Fit of System Dynamics Models, Dynamica, 10:2, 51-66.

Tustin A (1953) The Mechanism of Economic Systems, Harvard University Press, Cambridge, MA.

Warren K (2020) Engineering of Strategy: A General Unified Theory of Performance and Strategic

Management. In: System Dynamics: Theory and Applications, Dangerfield BC (Ed.), Springer, New York, 69-90.

Wolstenholme EF (2003) Towards the definition and use of a core set of archetypal structures in system dynamics, System Dynamics Review, 19:1, 7-26.

Wolstenholme EF \& McKelvie D (2019) The Dynamics of Care, Springer, New York. (A book which sets out many of the health \& social care models which the authors have developed during their careers as consultants.)

\section{AUTHOR BIOGRAPHY}

BRIAN DANGERFIELD has been teaching and researching using the system dynamics methodology for nearly fifty years. His career as an academic has involved spells in Business \& Management departments at the universities of Liverpool, Salford and Bristol. His research output has included a range of system dynamics applications in areas such as business, health, economic modelling and epidemiology. 